\title{
Anatomopathological Assessment of the Diaphragm in Formalin-Fixed, Paraffin-Embedded Sections
}

\section{Avaliação anatomopatológica do diafragma em cortes fixados em formalina e incorporados em parafina}

\author{
Ricardo Aparecido Baptista Nucci ${ }^{1,2}$ Wilson Jacob-Filho ${ }^{2,3}$ Alexandre Leopold Busse ${ }^{2,3}$ \\ Laura Beatriz Mesiano Maifrino ${ }^{4,5}$ Romeu Rodrigues de Souza ${ }^{6,7}$
}

\footnotetext{
1 Department of Pathology, Faculty of Medicine, Universidade de São Paulo, São Paulo, SP, Brazil

2 Postgraduate Pathology program, Faculty of Medicine, Universidade de São Paulo, São Paulo, SP, Brazil

3 Department of Geriatrics, Faculty of Medicine, Universidade de São Paulo, São Paulo, SP, Brazil

${ }^{4}$ Laboratory of Morphoquantitative Studies and Immunohistochemistry, Department of Physical Education, Universidade São Judas Tadeu, São Paulo, SP, Brazil

${ }^{5}$ Postgraduate Physical Education program, Universidade São Judas

Tadeu, São Paulo, SP, Brazil

${ }^{6}$ Department of Anatomy, Institute of Biomedical Sciences,

Universidade de São Paulo, São Paulo, SP, Brazil

7 Postgraduate Aging Sciences program, Universidade São Judas

Tadeu, São Paulo, SP, Brazil
}

J Morphol Sci 2018;35:173-176.

\begin{abstract}
Address for correspondence Ricardo Aparecido Baptista Nucci, PhD, Departamento de Patologia, Faculdade de Medicina da Universidade de São Paulo, Avenida Dr. Arnaldo, 455, 01246-903 São Paulo, SP, Brazil (e-mail: r.aparecido.nucci@uol.com.br).
\end{abstract}

Introduction The analysis of frozen muscle biopsies has become a routine method in the evaluation of muscle structure in health and disease. However, the technique for frozen muscle specimens is not widely available in countries with limited medical facilities. The present study aimed to elucidate a reproducible formalinfixed and paraffin-embedded (FFPE) method for this type of analysis in postmortem muscles.

Methods Diaphragm muscle was obtained within 1 hour of sudden death. Diaphragm strips were washed in saline solution, fixed in $10 \%$ formalin, frozen at $4^{\circ} \mathrm{C}$ in a refrigerator, and stored for 24 hours. Then, the tissue samples were processed into paraffin-embedded blocks. Transversal sections were cut from each paraffin block and stained with hematoxylin and eosin, Picrosirius red, Verhoeff-Van Gieson, and Congo red for the qualitative analysis.

Results Our analysis indicated a well-preserved muscle.

Conclusion In summary, we demonstrate a simple technique for a reproducible FFPE method in postmortem muscle tissues. received

February 12, 2018

accepted

August 9, 2018

published online

October 11, 2018
DOI https://doi.org/

10.1055/s-0038-1673611.

ISSN 2177-0298.
Copyright $\odot 2018$ by Thieme Revinter

Publicações Ltda, Rio de Janeiro, Brazil
License terms

(ㄷ) (i) $\ominus$ (\$) 


\section{Introduction}

The diaphragm (DIA) is the primary muscle of inspiration. Therefore, its uncompromised function is essential to support the ventilatory and gas exchange demands. ${ }^{1}$ Many patients with chronic obstructive pulmonary disease (COPD) or emphysema show high levels of DIA activity both at rest and during exercise, in which the DIA may experience maximal activation leading to irreversible muscle injury, mechanical failure, and death. ${ }^{1-3}$

The analysis of frozen muscle biopsies has become a routine method in the evaluation of muscle structure and function in health and disease. ${ }^{4,5}$ However, the analysis of frozen muscle specimens is not widely available in countries with limited medical facilities. ${ }^{6}$

Usually, postmortem studies use formalin-fixed and paraffin-embedded (FFPE) methods in the health sciences. ${ }^{7,8}$ Studies of postmortem bone, ${ }^{9}$ ligament, ${ }^{10,11}$ skin, ${ }^{12}$ articular cartilage, ${ }^{13}$ and spinal segments ${ }^{14}$ show relatively small variations from their live mechanical properties. Although many changes in skeletal muscle properties have been hypothesized in postmortem tissues, there is still limited quantitative and qualitative data available.

As the successful evaluation of postmortem muscle integrity in FFPE muscle sections has rarely been described, the present study aimed to elucidate a reproducible FFPE method for this type of analysis in postmortem DIA muscle.

\section{Materials and Methods}

Autopsy verification is mandatory in Brazil to define the cause of death for most individuals who die of natural causes. The São Paulo Autopsy Service (Serviço de Verificação de Óbito [SVO], in the Portuguese acronym) is the major morgue serving the metropolitan area of São Paulo, Brazil. ${ }^{8}$ The present study was approved by the ethical committee of the Faculdade de Medicina of Universidade de São Paulo under the registration number 2.209.383. The division of pathology from the Instituto do Coração (INCOR) provided the material for the present study exclusively for the sake of methodological standard in future projects.

\section{Muscle Samples}

Specimens from the right lobule ${ }^{15}$ of the DIA muscle was obtained within 1 hour of sudden death ${ }^{5,16}$ from a previously physically healthy 51-year-old man who died of myocardial infarction.

Diaphragm strips $(\sim 5 \times 5 \times 5 \mathrm{~mm})$ were removed at 4 to 6 $\mathrm{cm}$ from the central tendon (midcostal) to avoid the muscle fibers that radiate toward this tendon insert. ${ }^{1,15}$ The DIA strips were gently washed in saline solution, placed in a standard histological cassette (SWINGSETTE ${ }^{\mathrm{TM}}$ Tissue Processing/ Embedding Cassettes, Histocell Soluções em Anatomia Patológica Ltda., São Paulo, SP, Brazil) to re-establish the initial length, and immediately fixed in $10 \%$ formalin, frozen at $4^{\circ} \mathrm{C}$ in a refrigerator, and stored for 24 hours. ${ }^{5,16}$ Then, the tissue samples were processed into paraffin-embedded blocks. ${ }^{6}$

\section{Histological Staining}

Transversal sections of $6 \mu \mathrm{m}$ thick ${ }^{15}$ were cut from each paraffin block for histological staining. The sections were stained with the hematoxylin and eosin (H\&E) standard method for the general structure of the sample. ${ }^{4}$ The sections were also stained with standard methods ${ }^{4,17}$ for: (a) Picrosirius red (collagen fibers); (b) Verhoeff-Van Gieson (elastic fibers); and (c) Congo red (amyloid deposits).

The qualitative evaluation of the tissue was performed by photographing 40 fields selected at random for each staining technique. The images were captured using a Zeiss Microscope (Binocular microscope Axio Lab.A1 with phototube, Carl Zeiss, Thornwood, NY, USA) with the specific software AxioVision, Version 4.8 (Zeiss, Thornwood, NY, USA).

\section{Results}

The H\&E stain positivity indicated a well-preserved muscle ( - Fig. 1A). The Picrosirius red stain gave superior results under a polarized filter (- Fig. 1B), in which different collagen fiber types were observed in the endomysium, in the perimysium, and in the epimysium. Additionally, we could observe a weak positive stain for elastic fibers ( - Fig. 2A), and amyloid (-Fig. 2B).

\section{Discussion}

With death, a series of postmortem events initiate, including the loss of enzyme activities. ${ }^{5}$ The reliability of postmortem muscle samples depends on the extent of these cellular alterations.

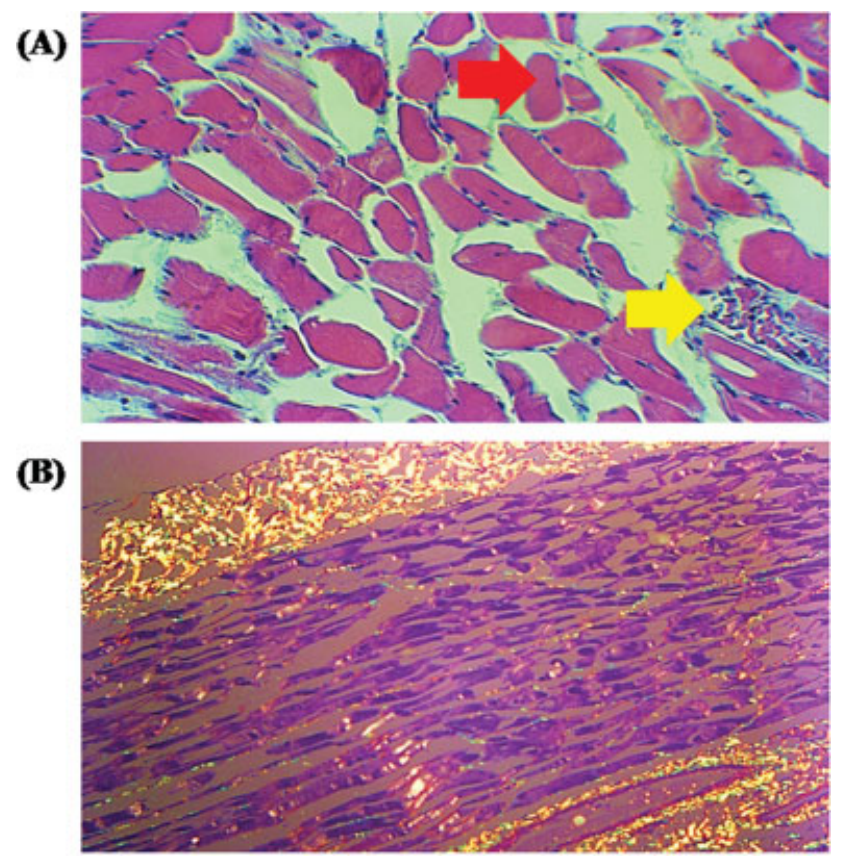

Fig. 1 Cross-sectional images of the diaphragm (DIA) muscle stained with hematoxylin and eosin $(A)$ or picrosirius red $(B)$ (100x magnification). A) Red arrow: muscle fiber; yellow arrow: inflammatory process. (B) Picrosirius red stain in polarized microscopy showing type I collagen fibers (red); intermediate fibers (yellow/orange); and type III collagen fibers (green). 
(A)

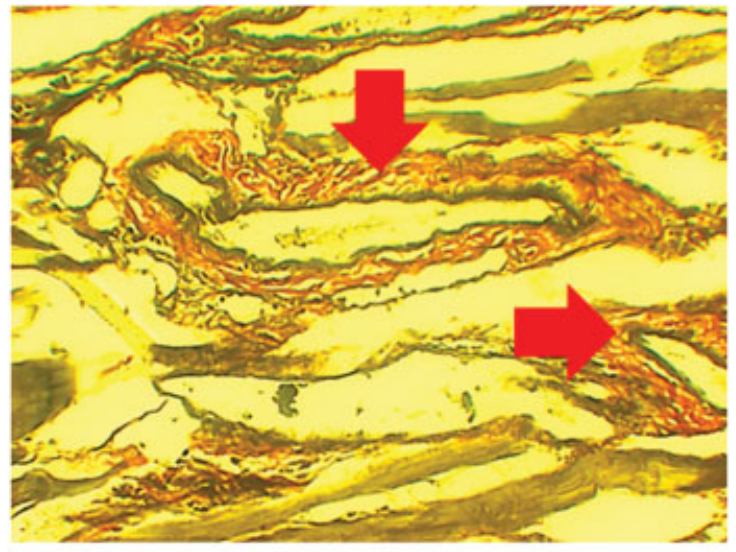

(B)

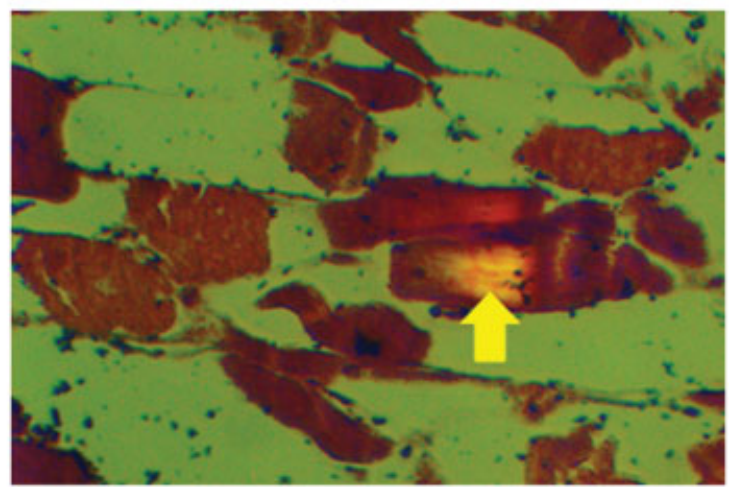

Fig. 2 Cross-sectional images of the diaphragm (DIA) muscle stained with the Verhoeff-Van Gieson (A) or Congo red (B) (400x magnification). A) Red arrows: elastic fibers. B) Congo red stain in polarized microscopy showing amyloid (yellow arrow).

Eriksson et $\mathrm{al}^{5}$ demonstrated that muscle samples stored for a maximum of 10 days in controlled temperature $\left(4^{\circ} \mathrm{C}\right)$ are reliable as nitrogen stored samples. Van Ee et $\mathrm{al}^{16}$ had similar results, indicating that lower temperatures may maintain the integrity of the muscle fibers. We have frozen the samples at $4^{\circ} \mathrm{C}$, which maintained its integrity.

Nevertheless, it is reported that muscles stiffen as they enter rigor mortis. ${ }^{18}$ An experimental study by Fitzgerald ${ }^{19}$ reported that the elastic compliance decreased $95 \%$, and that the viscous compliance decreased $98.3 \%$ in relation to the living muscle tissue values at $\sim 6.5$ hours after death. Van Ee et $\mathrm{al}^{16}$ reported that a period of 0.5 hours postmortem (prerigor) had only a modest effect on the mechanical properties of the muscle, while at 48 hours postmortem (postrigor), the response changed greatly. We have chosen to freeze the samples 1 hour postmortem to maintain the prerigor integrity.

A pilot study of an autolytic change in rat diaphragms demonstrated that, up to 96 hours postmortem, the only postmortem morphological artifact was a decrease in the intensity of the H\&E staining. ${ }^{20}$ This artifact was not observed in the present study. The Picrosirius red stain was successful because it specifically labels collagen molecules without relying on the recognition of antigens, which may be degraded postmortem or during fixation. ${ }^{15,17}$ Although we have observed a decrease in the intensity of the Verhoeff-Van Gieson staining, Rodrigues et $\mathrm{al}^{21}$ observed that the viscoelastic properties of the DIA decreases with aging, which may explain our finding. Finally, the Congo red staining was efficient to demonstrate amyloid deposits, as has been shown by previous studies with FFPE in the nervous system. ${ }^{22,23}$

Recently, Suriyonplengsaeng et $\mathrm{al}^{6}$ successfully developed an immunohistochemistry (IHC) technique, with heatmediated antigen retrieval, for FFPE muscle biopsy specimens. This study may encourage more researches to use IHC on FFPE muscles in autopsy studies as well.

The present study has some weaknesses that need to be acknowledged for proper interpretation. The main limitation of the present study is the small sample size, which was limited to one patient. However, the small sample size would not explain our positive findings. Due to the limited literature, we could not carry out an extensive discussion about our findings. Nevertheless, we have highlighted in the present study a simple and valid methodological approach for the analysis of postmortem muscle that could be improved and discussed in depth in further studies.

\section{Conclusion}

In summary, we demonstrate a simple and reproducible technique for FFPE in postmortem tissue. We suggest that this method could become a valuable tool for the diagnosis of anatomopathological changes in the DIA muscle.

\section{Acknowledgments}

This study was financed in part by the Coordenação de Aperfeiçoamento de Pessoal de Nível Superior (CAPES, in the Portuguese acronym) Finance Code 001. We thank the SVO of the department of pathology of Faculdade de Medicina da Universidade de São Paulo, and Dr. Laura Beatriz Mesiano Maifrino for the technical support.

\section{References}

1 Poole DC, Sexton WL, Farkas GA, Powers SK, Reid MB. Diaphragm structure and function in health and disease. Med Sci Sports Exerc 1997;29(06):738-754

2 Sinderby C, Beck J, Spahija J, Weinberg J, Grassino A. Voluntary activation of the human diaphragm in health and disease. J Appl Physiol (1985) 1998;85(06):2146-2158

3 Topeli A, Laghi F, Tobin MJ. The voluntary drive to breathe is not decreased in hypercapnic patients with severe COPD. Eur Respir J 2001;18(01):53-60

4 Dubowitz V, Oldfors A, Sewry CA. Muscle Biopsy E-Book: A Practical Approach. Elsevier Health Sciences; 2013

5 Eriksson O, Eriksson A, Ringqvist M, Thornell LE. The reliability of histochemical fibre typing of human necropsy muscles. Histochemistry 1980;65(03):193-205

6 Suriyonplengsaeng C, Dejthevaporn C, Khongkhatithum C, et al. Immunohistochemistry of sarcolemmal membrane-associated proteins in formalin-fixed and paraffin-embedded skeletal muscle tissue: a promising tool for the diagnostic evaluation of common muscular dystrophies. Diagn Pathol 2017;12(01):19

7 Farfel JM, Nitrini R, Suemoto CK, et al; Brazilian Aging Brain Study Group. Very low levels of education and cognitive reserve: a clinicopathologic study. Neurology 2013;81(07):650-657

8 Suemoto CK, Ferretti-Rebustini RE, Rodriguez RD, et al. Neuropathological diagnoses and clinical correlates in older adults in Brazil: A cross-sectional study. PLoS Med 2017;14(03):e1002267

9 Borchers RE, Gibson LJ, Burchardt H, Hayes WC. Effects of selected thermal variables on the mechanical properties of trabecular bone. Biomaterials 1995;16(07):545-551 
10 Smith CW, Young IS, Kearney JN. Mechanical properties of tendons: changes with sterilization and preservation. J Biomech Eng 1996;118(01):56-61

11 Turner WD, Vasseur P, Gorek JE, Rodrigo JJ, Wedell JR. An in vitro study of the structural properties of deep-frozen versus freezedried, ethylene oxide-sterilized canine anterior cruciate ligament bone-ligament-bone preparations. Clin Orthop Relat Res 1988; (230):251-256

12 Foutz TL, Stone EA, Abrams CF Jr. Effects of freezing on mechanical properties of rat skin. Am J Vet Res 1992;53(05): 788-792

13 Kiefer GN, Sundby K, McAllister D, et al. The effect of cryopreservation on the biomechanical behavior of bovine articular cartilage. J Orthop Res 1989;7(04):494-501

14 Callaghan JP, McGill SM. Frozen storage increases the ultimate compressive load of porcine vertebrae. J Orthop Res 1995;13(05): 809-812

15 Scott A, Wang X, Road JD, Reid WD. Increased injury and intramuscular collagen of the diaphragm in COPD: autopsy observations. Eur Respir J 2006;27(01):51-59

16 Van Ee CA, Chasse AL, Myers BS. Quantifying skeletal muscle properties in cadaveric test specimens: effects of mechanical loading, postmortem time, and freezer storage. J Biomech Eng 2000;122(01):9-14

17 Junqueira LCU, Bignolas G, Brentani RR. A simple and sensitive method for the quantitative estimation of collagen. Anal Biochem 1979;94(01):96-99

18 Mayer RG, Reed JD. Embalming: history, theory, and practice. Norwalk, CT: Appleton \& Lange; 1990

19 Fitzgerald ER. Dynamic mechanical measurements during the life to death transition in animal tissues. Biorheology 1975;12(06): 397-408

20 Macgowan NA. Diaphragm injury in chronic respiratory disease. MSc thesis. Rehabilitation Sciences, University of British Columbia, Vancouver, Canada, 1998

21 Rodrigues CJ, Rodrigues Junior AJ. A comparative study of aging of the elastic fiber system of the diaphragm and the rectus abdominis muscles in rats. Braz J Med Biol Res 2000;33(12):1449-1454

22 Askanas V, Engel WK, Alvarez RB. Enhanced detection of congo. -redpositive amyloid deposits in muscle fibers of inclusion body myositis and brain of Alzheimer's disease using fluorescence technique. Neurology 1993;43(06):1265

23 LaFerla FM, Green KN, Oddo S. Intracellular amyloid- $\beta$ in Alzheimer's disease. Nat Rev Neurosci 2007;8(07):499-509 